\title{
Zur Einzelsprachenspezifik wissenschaftlichen Sprachausbaus im gnoseologischen Funktionsbereich von Sprache
}

\author{
Winfried Thielmann (Chemnitz)
}

\begin{abstract}
This paper attempts to demonstrate that academic research depends, to a certain degree, on the language in which it is conducted. The reason for this is that European languages have, due to their typological differences, different histories of linguistic expansion (Sprachausbau). As can be shown for German and English, the purpose of naming the object of investigation in academic research is served by linguistic devices of very different nature. This may lead to different conceptions of the object of research and thus impact on the course of investigation.
\end{abstract}

\section{Vorbemerkungen}

Wissenschaft ist sprachgebunden. Auch die in vielen Wissenschaften benutzten mathematischen Kalküle sind aus in Sprache gefassten Überlegungen erwachsen und funktionieren nur aufgrund ihrer prinzipiellen Rückführbarkeit auf Sprache. ${ }^{1}$

Als sprachabhängige Unternehmung zehrt Wissenschaft wesentlich von den Möglichkeiten der je spezifischen Einzelsprachen, derer sie sich bedient. Hieraus ergibt sich im Interesse der wissenschaftlichen Innovation das Erfordernis einer komparativen Untersuchung dieser Möglichkeiten (vgl. Ehlich 1995).

Wissenschaft nimmt diese Möglichkeiten für wissenschaftsspezifische Zwecke in den Dienst: das Auffinden neuer Erkenntnis, das Fixieren neuer Erkenntnis und das Kommunizieren und Durchsetzen der Innovation in der Community. Für das Auffinden, Fixieren und Kommunizieren neuer Erkenntnis wird ein prinzipieller Funktionsbereich aller Sprachen relevant - der gnoseologische (Ehlich 1998 ND 2007: 159):

Die Wahrnehmung, Verarbeitung und die Entwicklung von Wissen über die Welt und über sich selbst ist bei der Gattung Mensch unlösbar mit Sprache verbunden.

Die Mittel, die Einzelsprachen für den gnoseologischen Funktionsbereich vorhalten, sind wesentlich solche, mit denen Wissenskomplexe objektiviert vorgehalten werden können (vgl. ebd.) - Ausdrücke des Symbolfelds (Bühler 1934). Ihre sprachspezifische Ausgestaltung ist in hohem Maße sprachtypabhängig:

Die Konkretisierungen der gnoseologischen Funktion zeigen die Gattung in der Vielfalt eines menschheitsübergreifenden Experiments. Das Medium Sprache hat sich in unterschiedlichsten Sprachtypen konkretisiert. Die Typologie der menschlichen Sprachen kann verstanden werden als Menge der stabilisierten Problemlösungen in Bezug auf die Erkenntnisstiftung im Medium Sprache. (Ehlich 1998 ND 2007: 159)

Für die Wissenschaftssprachkomparatistik ergibt sich somit die Notwendigkeit, allgemein die einzelsprachenspezifische Ausprägung dieser Mittel vergleichend herauszuarbeiten und ihre

\footnotetext{
${ }^{1} \mathrm{Zu}$ den geschichtlichen Voraussetzungen der Kalkülisierung vgl. Maier (1955), Simonyi (1990) und Thielmann
} (1999). 
wissenschaftliche In-den-Dienst-Nahme anhand des wissenschaftlichen Sprachausbaus nachzuvollziehen.

Dies möchte ich hier am Beispiel deutscher und englischer Wissenschaftssprache versuchen. Ich betrachte daher zunächst allgemein die Ausprägung des Symbolfelds im Deutschen und Englischen und gehe anschließend auf die wissenschaftliche Ausbaugeschichte beider Sprachen ein. Auf dieser Basis diskutiere ich anhand von aktuellen Paralleltexten die Konsequenzen für den gnoseologischen Funktionsbereich in der Wissenschaft.

\section{Symbolfeldausdrücke im Englischen und im Deutschen}

Das sprachliche Kommunizieren von Wissenskomplexen ist auf sprachliche Mittel angewiesen, die zweierlei leisten: Der Wissenskomplex muss benannt werden können, und er muss als Wissenskomplex benannt werden können. Der Zweck des nennenden Sistierens von Wissenskomplexen, der Herstellung begrifflicher Nennqualität, wird in typologisch verschiedenen Sprachen erwartbar verschieden bearbeitet, wie ich im folgenden anhand des Deutschen und Englischen zeigen möchte. Die Betrachtung erfolgt im Rahmen funktional-pragmatischer Theoriebildung; insbesondere wird von Ehlichs Unterscheidung zwischen Sprachmitteln und Sprachzwecken (1981) Gebrauch gemacht.

Im Deutschen stehen für den Zweck des nennenden Sistierens von Wissenskomplexen Ausdrücke zur Verfügung, die einer spezifischen Wortart zugehören: Substantive. Substantive sind im Deutschen durch die grammatische Eigenschaft des Genus gekennzeichnet. Nur Ausdrücke mit Genus sind determinationsfähig; Ausdrücke ohne Genus erfahren bei Determination eine Feldtransposition (das Ich) (vgl. Ehlich 1986) oder eine feldinterne Transposition (das Wahre) (vgl. Redder 2005). Das Genus des Determinators wird vom Substantiv regiert. Bei Feldtranspositionen oder feldinternen Transpositionen wird hingegen das Neutrum als Default-Genus über den Determinator zugewiesen. Stoff- und Eigennamen treten i. d. R. ohne Determinator auf; Appellativa erfordern in den meisten sprachlichen Handlungszusammenhängen Determination, wenn sie auch in bestimmten (Sub-)Textarten, so z.B. Listen und Überschriften, isoliert auftreten können:

$$
\begin{array}{ll}
\text { Bild (Öl, ca. 1832; Schätzwert 1200,-) } & \text { (Auktionskatalog) } \\
\text { Turnhalle eingestürzt } & \text { (Zeitungsüberschrift) }
\end{array}
$$

Neben dem Artikelsystem (bestimmter Art./unbest. Art./Nullartikel) können weitere Ausdrücke und Wortformen quasi-determinative Aufgaben übernehmen (vgl. Zifonun/Hoffmann/Strecker 1997: 1926-1950; Hoffmann 2003: 61-64), so z. B. attributive Deixis und Phorik (mein Auto, ihre Tasche), Quantoren (keine Semmeln, alle Leute, jedes Buch) oder das pränominale Genitivattribut (Peters Auto).

Substantive erscheinen so im Deutschen prototypisch als strukturelle Zentren von Wortgruppen, als Köpfe von Nominalphrasen (ausführl. z. B. Zifonun/Hoffmann/Strecker 1997: 1926-1950), die Satzgliedstatus (Subjekt, direktes Objekt, Prädikativ etc.) besitzen können oder als Attribute fungieren (z.B. Genitivattribut). Ferner bilden Nominalphrasen mit Präpositionen Präpositionalphrasen, die als Präpositionalobjekte (Er hat sich über den Film geärgert.), Adverbiale (Der Umsatz ist im letzten Jahr gesunken.) oder Präpositionalattribute (Angst vor der Prüfung) auftreten.

Aufgrund der Rektionsbeziehungen zwischen Substantiv und Determinator sowie der Flexion adjektivischer oder partizipialer Attribute besteht auch innerhalb komplexer Nominalphrasen ein starker struktureller Zusammenhalt, weswegen Weinrich (2003) von Nominalklammer spricht: 


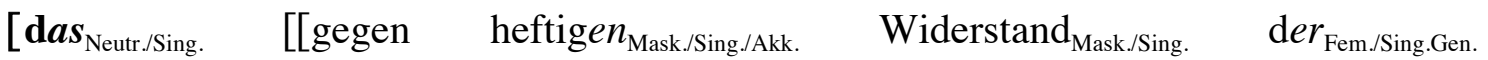

$$
\begin{aligned}
& \text { Opposition } \left.\left.\left._{\mathrm{Fem} . / \mathrm{Sing} .}\right]_{\mathrm{PP}(\mathrm{Adv} .)} \text { verabschiedet } e\right]_{\text {Part.Attr. }} \text { Gesetz }_{\text {Neutr./Sing. }}\right]_{\mathrm{NP}}
\end{aligned}
$$

Durch den Determinator das wird eine Hörer/Leser-Erwartung auf einen mit ihm hinsichtlich Genus und Numerus kongruierenden nominalen Kopf ausgelöst. Dass direkt nach dem Determinator die Präposition gegen auftritt, bedeutet für die hörerseitige Vorauskonstruktion, dass zunächst eine als Präpositionalphrase realisierte adverbiale Bestimmung oder Ergänzung zu einem attributiven Partizip zu verarbeiten ist.

Die Nominalphrasenstruktur im Deutschen wird mithin wesentlich dadurch bestimmt, dass ein genushaltiger Symbolfeldausdruck, also ein Substantiv, das strukturelle Zentrum bildet. Weitere an der Nominalphrasenbildung beteiligte Wortarten und -formen des Symbolfeldes (z.B. Adjektive und Partizipien) sind durch ihre Flexionscharakteristika gekennzeichnet und hinsichtlich ihres syntaktischen Status ausgewiesen (z.B. als Attribut). Für den Hörer/Leser ist also bei der Verarbeitung einer deutschen Nominalphrase klar, wie die beteiligten Symbolfeldausdrücke im ontologischen Sinne benennen und in welcher syntaktischen Beziehung sie zueinander stehen.

Im Englischen, einer weitgehend isolierenden Sprache ${ }^{2}$, liegen die Verhältnisse völlig anders. Im Gegensatz zum Deutschen gibt es kaum grammatische Indikatoren dafür, wie ein Symbolfeldausdruck (im ontologischen Sinne) benennt. Hauptwortarten gibt es nicht. ${ }^{3}$ Die puren Symbolfeldausdrücke (Redder 2005) erfahren ihre ontologische Vereindeutigung daher über die Phrasenstrukturen, die vom Hörer/Leser wiederum auf Basis seines Wissens über Abfolgeregularitäten von Satzgliedern sowie semantische Kompatibilität zu kombinieren sind:

(3) The beautiful act selfishly.

(3') The beautiful act of random kindness occurred when an elderly gentleman pulled up (...). (Internet-Beleg)

(4) He's the boss.

(4') Don't boss me around!

In (3) ist der pure Symbolfeldausdruck beautiful Kopf der Nominalphrase in Subjektposition, da nach ihm - am Adverbial erkennbar - ein Prädikat auftritt: act selfishly. Der ontologische Status der durch beautiful und act symbolisierten Gehalte (abstrakter Gegenstand/Eigenschaft/Handlung) ist für den Hörer/Leser aus seinem (impliziten) Wissen über Satzgliedabfolgen zu rekonstruieren. In (3') ergibt sich für den Hörer/Leser der attributive Status von beautiful retrospektive daraus, dass act aufgrund des folgenden Präpositionalattributs Kopf der Nominalphrase sein muss und daher nicht Prädikat sein kann. Dass boss in (4) Kopf einer Nominalphrase ist, wird daraus ersichtlich, dass nach is ein Determinator auftritt, wodurch ein als Nominalphrase realisiertes Prädikativ angekündigt wird. In (4') ist boss hingegen Teil des Prädikats, da die Verbform don't vorhergeht.

Feldtranspositionen gegenüber erweist sich das Englische als äußerst resistent, wie z.B. die Übersetzung der Werke Freuds zeigt (z.B. das Ich - the ego; vgl. Kuenkamp 1995). So sind auch die kühnen Feldtranspositionen des Lyrikers Gerard Manley Hopkins (1844-1889) in den Ausgaben seiner Lyrik nach wie vor erläuterungsbedürftig. Self, eine zumindest paraoperative Prozedur, erfährt hier durch Einsetzung in den "Prädikats-Slot" eine Feldtransposition ins Symbolfeld (selves):

\footnotetext{
${ }^{2} \mathrm{Zu}$ den sprachlichen Entwicklungsprozessen vgl. Blake (1992).

3 "(...) many lexemes are now 'underspecified' for 'word classes'; moreover, the specification takes place on the syntactic level by phrase markers, e.g. articles." (Vogel 2000: 274)
} 
(...) Each mortal thing does one thing and the same:

Deals out that being indoors each one dwells;

Selves - goes itself; myself it speaks and spells; (...)

(G. M. Hopkins: As Kingfishers Catch Fire, ca. 1881 (aus: Hopkins 1961, Hervorhebung i. O.)

Sehr viel elastischer verhält sich das Englische jedoch gegenüber der Besetzung syntaktischer slots mit fremdem Wortmaterial. Dieses Verfahren, mit dem Burgess in "A Clockwork Orange" (1962) das Jugend-Idiom "Nadsat" schuf, hat mit zu der fulminanten Rezeptionsgeschichte des Werks beigetragen:

'Oh, you've recovered consciousness.'

That was like a big rotful for a malenky ptitsa like her, and I tried to say so, but the slovos came out only like er er er. She ittied off and left me on my oddy knocky, and I could viddy now that I was in a malenky room of my own, not in one of these long wards like I had been in as a very little malchik, full of coughing dying starry vecks all around to make you want to get well and fit again. It had been like diphtheria I had had then, O my brothers. (Burgess 2000: 126)

Zusammenfassend: Im Deutschen gibt es Ausdrücke, die bereits eine begriffliche Nennqualität besitzen: Substantive. Diese sind im einfachen Fall genushaltige Stämme, die (von Stoffund Eigennamen abgesehen) mit Determinatoren und quasi-determinativen Ausdrücken sowie attributiven Zusätzen satzgliedfähige Phrasen bilden. Im genuslosen Englischen gibt es keine Ausdrücke, die von sich aus über begriffliche Nennqualität verfügen. Es liegen dort im wesentlichen pure Symbolfeldausdrücke im Sinne von Redder (2005) vor. Diese erhalten substantivische Qualität (d.h. ontologische Vereindeutigung des durch sie benannten Gehaltes) über Phrasenstrukturen, die ihrerseits vom Hörer/Leser anhand impliziten Wissens über die Satzgliedabfolge und semantische Kompatibilität der beteiligten Symbolfeldausdrücke zu rekonstruieren sind. Feldtranspositionen gegenüber erweist sich das Englische als außerordentlich resistent.

Wie sich im folgenden zeigen wird, ist die wissenschaftliche Ausbaugeschichte des Deutschen und Englischen auch im Zusammenhang der zwischen den Sprachen bestehenden typologischen Gegensätze zu sehen.

\section{Wissenschaftlicher Sprachausbau des Englischen und Deutschen}

Mit dem Beginn des 17. Jahrhunderts tritt in Europa eine wissenschaftssprachgeschichtlich höchst bedeutsame Entwicklung auf: Hatte man sich vorher einer wissenschaftlichen "Universalsprache" bedient, nämlich des Lateinischen, so begannen vor allem die Vertreter der modernen Naturwissenschaft, zunehmend ihre Muttersprachen bei der Formulierung und Verbreitung neuer wissenschaftlicher Erkenntnis zu gebrauchen. Die Gründe für diesen Prozess lassen sich m. E. recht deutlich an Textbeispielen von Isaac Newton zeigen. Newton hatte 1687 sein Hauptwerk über Mechanik auf Lateinisch veröffentlicht. Der Text ist praktisch durchgängig in der Form Definition - Satz - Beweis gehalten. Wir betrachten einen Teil der ersten Definition: 


\section{DEFINITIO I.}

Quantitas materiae est mensura eiusdem orta ex illius densitate et magnitudine conjunctim.

Aer densitate duplicata, in spatio etiam duplicato, fit quadruplus; in triplicato sextuplus. Idem intellege de nive \& pulveribus per compressionem vel liquefactionem condensatis. (...) (Newton 1972: 39f)

1. Definition: Die Stoffmenge ist das Maß derselben, welches aus dem Produkt ihrer Dichte und Größe entsteht.

Luft von verdoppelter Dichte wird bei verdoppeltem Raum das Vierfache; in verdreifachtem Raum das Sechsfache. Dasselbe erkennt man bei Schnee und verschiedenen Arten von Staub, die durch Zusammendrücken oder Verflüssigung verdichtet wurden. (Übers. W.T.)

Man sieht, dass Newton die Masse (quantitas materiae) unter Rekurs auf experimentelle Befunde fasst. Zugleich macht das idem intellege! - also etwa: "sieh analog hierzu ein!" - eine durchaus apodiktische Grundhaltung deutlich, wie sie für Texte scholastischer Tradition nicht unüblich war.

Im Jahre 1704 veröffentlicht Newton seine Monographie Opticks auf Englisch, woraus hier die zweite Definition angeführt ist (Hervorhebungen in Fettdruck W.T.):

\section{DEFIN. II}

Refrangibility of the Rays of Light, is their Disposition to be refracted or turned out of their Way in passing out of one transparent Body or Medium into another. And a greater or less Refrangibility of Rays, is their Disposition to be turned more or less out of their Way in like Incidences on the same Medium. Mathematicians usually consider the Rays of Light to be Lines reaching from the luminous Body to the body illuminated, and the refraction of those Rays to be the bending or breaking of those Lines in their passing out of one Medium into another. And thus may Rays and Refractions be considered, if Light be propagated in an instant. But by an Argument taken from the Æquations of the times of the Eclipses of Jupiter's Satellites it seems that Light is propagated in time, spending in its passage from the Sun to us about Seven Minutes of time: And therefore I have chosen to define Rays and Refractions in such general terms as may agree to Light in both cases. (Newton 1704: 2)

Man sieht sofort einen massiven Unterschied zu den Principia: In dem englischen Text passiert etwas völlig anderes als in dem lateinischen. Wir finden Formulierungen vor, mit denen Newton explizit auf den Wissensstand und die Auffassungen der wissenschaftlichen Community Bezug nimmt: usually consider, may be considered, by an argument taken from, it seems that, I have chosen to define, in such general terms as may agree to. Dass Newton so vorgeht, hängt damit zusammen, dass es bezüglich der Ausbreitung des Lichtes zwei konkurrierende Auffassungen gab. Die einen waren der Auffassung, dass Licht sich instantan ausbreitet; andere hatten bereits astronomische Evidenz dafür akzeptiert, dass Licht etwa sieben Minuten von der Sonne zur Erde unterwegs ist. Um beide Gruppen sozusagen bedienen zu können, definiert Newton Lichtstrahlen und ihre Brechung so, dass seine Definition sich mit beiden Auffassungen verträgt.

Man sieht hier deutlich, dass der Wissenschaftler nicht mehr, wie dies noch in den Principia der Fall war, neues Wissen einfach vorträgt, sondern dass er dieses neue Wissen zugleich auf 
den Kenntnisstand der Community bezieht. Mit anderen Worten: Newton konzipiert seinen Text auf eine Situation hin, in der neues Wissen prinzipiell strittig ist. Die sprachlichen Mittel, mit denen die Strittigkeit des neuen Wissens bearbeitet wird, nenne ich mit Bezug auf Ehlich (1993), wo sie zum ersten Mal beschrieben wurden, eristische Strukturen.

Wir sehen also folgendes: Newton übernimmt einen großen Teil der Terminologie aus dem Lateinischen: refrangibility, disposition, medium, incidence, refraction. Die eristischen Strukturen sind hingegen gemeinsprachlicher Natur.

Zwei Jahre später ließ Newton die Opticks ins Lateinische übersetzen - wohl, um ihnen noch weitere Verbreitung zu ermöglichen. Der Übersetzer versichert im Vorwort, dass er sich exakt an die Vorlage gehalten habe und dass Newton persönlich die Übersetzung autorisiert hätte. Wir betrachten nun die lateinische Übersetzung der englischen Definition (Hervorhebungen im Fettdruck W.T.):

\section{DEFIN. II}

Refrangibilitas radiorum Luminis, est dispositio ea, qua ita comparati sunt, ut in transeundo ex uno Corpore translucido, sive Medio, in aliud, refringantur seu de via deflectantur. Et major minorve Radiorum Refrangibilitas, est Dispositio ea qua apti sunt, ut in paribus incidentiis super unum idemq; Medium, magis minusve de via detorqueantur. Mathematici plerunq; secum ita fingunt; radios Luminis esse lineas a corpore Lucido ad corpus illuminatum pertingentes ; horumq; radiorum Refractionem, esse Linearum istarum Flexionem aut Fractionem in transeundo ex uno Medio in aliud. Atq; hoc quidem de Radiis \& Refractionibus dici possit; si Lumen uno momento propagetur. Verum cum ex temporum Eclipsium Jovis Satellitum Æquationibus, id colligi videatur, Lumen spatio temporis propagari ; adeo ut e Sole in Terram septem circiter minutorum spatio deferatur ; Radios ac Refractiones ita laxe definire malui, ut quicquid de Luminis propagatione statuatur, hæ tamen Definitiones in utramq; partem veræ ac certæ sint. (Newton 1706: 2)

Man sieht: Die propositionalen Gehalte des englischen Textes sind exakt wiedergegeben. Ein massives Problem hatte der Übersetzer aber offensichtlich genau mit denjenigen aus der Gemeinsprache geschöpften Formulierungen, mit denen die Strittigkeit von Wissen bearbeitet wird: Aus dem eleganten mathematicians usually consider ist ein mathematici plerunque secum ita fingunt geworden - also etwa: "Mathematiker stellen sich das häufig so vor". Aus may be considered wird dici possit - hier ist der mentale Bezug von consider völlig weg. Aus dem begründeten Darstellungsschritt therefore I have chosen wird malui, also schlichte Präferenz.

Solche Befunde sowie ähnliche Beobachtungen an Texten Galileis (Thielmann 2010) machen m. E. folgende Überlegung plausibel: Das Latein der Scholastik war für die Zwecke einer autoritätsbasierten Wissenschaft optimal ausgebaut. Die mit Galilei beginnende moderne Naturwissenschaft verlangt aber sprachliche Strategien der intersubjektiven Wahrnehmungs- und Wissensbearbeitung, für die das Latein der Scholastik keine Ressourcen bereithielt. Um der empirischen Forschung willen und derer, die sie tragen, büßt die Wissenschaft ihre Universalsprache ein, indem die Wissenschaftler auf Sprachen ausweichen, in denen die Basis für solche Mittel gesamtgesellschaftlich vorgehalten wird.

Zugleich sieht man anhand der lateinischen Übersetzung der Opticks deutlich, was 1706 aus dem Lateinischen, der ehemaligen wissenschaftlichen Universalsprache, geworden ist - eine lingua franca, die genau dort nicht präzise ist, wo die moderne Wissenschaft höchste Präzi- 
sion erfordert: bei der sprachlichen Bearbeitung der Strittigkeit neuen wissenschaftlichen Wissens. ${ }^{4}$

Der Ausbau des Englischen zur Wissenschaftssprache ereignet sich also zunächst einmal gerade nicht in demjenigen Bereich, den man gerne als hierfür zentral ansieht - dem terminologischen. Vielmehr ereignet er sich in dem demgegenüber unauffälligen, da weitgehend gemeinsprachlich erscheinenden, Bereich der eristischen Strukturen. Wie wir gesehen haben, erfahren hierbei gemeinsprachliche Mittel eine Zubereitung für die wissenschaftstypischen Zwecke der intersubjektiven Wahrnehmungs- und Wissensbearbeitung. Die für die Zwecke der Eristik funktionalisierten gemeinsprachlichen Ressourcen sind genuine Bestandteile der wissenschaftlichen Varietät, präzise Instrumente, die im Rahmen des wissenschaftlichen Geschäftes auch einen virtuosen Nutzer erfordern.

Mit Bezug auf das Englische, Italienische und Französische lässt sich sagen, dass diese Volkssprachen für die Wissenschaftler attraktiv wurden, weil sie über gesamtgesellschaftlich vorgehaltene sprachliche Ressourcen für den wissenschaftlichen Streit verfügten. In terminologischer Hinsicht werden hingegen nach wie vor vorwiegend lateinische Ressourcen genutzt, wie dies bei diesen Sprachen auch naheliegt.

Völlig anders stellt sich hingegen die Situation für das Deutsche dar. Der Ausbau des Deutschen zur Wissenschaftssprache vollzog sich - wohl auch bedingt durch den 30jährigen Krieg - mit erheblicher Verzögerung. Er erfolgte nicht von naturwissenschaftlicher, sondern wesentlich von philosophischer Seite her, und zwar im Rahmen des Programms der Aufklärung. Meine Ausführungen hierzu basieren wesentlich auf einem höchst instruktiven Aufsatz von Ulrich Ricken (1995). Dieser Aufsatz erhellt das sprachschöpferische Verdienst eines Mannes, dessen Name heute fast nur noch Spezialisten bekannt ist: des Philosophen Christian Wolff. Wolff, einem überzeugten Aufklärer, war es ein Anliegen, das Wissen seiner Zeit auch einem Publikum zugänglich zu machen, das des Lateinischen nicht mächtig war. Während nun im Italienischen, Französischen oder Englischen ein lateinisches Wort immer auch potentiell ein italienisches, französisches oder englisches ist, ist das im Deutschen nicht so. Es ist daher nur konsequent, dass Wolffs Arbeit an der deutschen Wissenschaftssprache im terminologischen Bereich ansetzt. Hierzu geht er nach folgendem Verfahren vor:

Dass ich die deutschen Wörter in ihrer ordentlichen Bedeutung nähme und darinnen den Grund der Benennung zu dem Kunst-Worte suchte. Denn auf solche Weise ist mein Kunst-Wort rein deutsch, weil ich deutsche Wörter in ihrer eigentlichen Bedeutung brauche und indem ich sie zu einem Kunst-Worte mache, auf Sachen ziehe, darinnen etwas anzutreffen so durch das Wort in seinem eigenen Verstande genommen angedeutet wird. (1726, zitiert nach Ricken 1995: 60)

Für die zeitgenössischen Wissenschaftler und Philosophen, die nur die gräko-lateinischen Termini der scholastischen Wissenschaftssprache kennen, muss Wolff, um sich verständlich zu machen, noch eigene Listen mit Worterklärungen hinzufügen. Betrachten wir einmal einige von Wolffs deutschen terminologischen Neuprägungen:

\footnotetext{
${ }^{4}$ Um möglichen Missverständnissen vorzubeugen: Die hier vorgetragene These besagt natürlich nicht, dass sich das Lateinische grundsätzlich nicht für neuzeitliche Wissenschaft eigne. Jedoch handelte es sich bei der den Pionieren der neuzeitlichen Naturwissenschaft vorfindlichen wissenschaftlichen Varietät des Lateinischen um eine Sprachlichkeit, die wesentlich aus der wissenschaftlichen Befassung mit autoritativen Texten hervorgegangen war. Diese Varietät war für die Wissensweitergabe, also hinsichtlich der terminologischen und propositionalen Dimension, hervorragend ausgebaut, wie man dies auch an den lateinischen Texten von Galilei und Newton sieht. Sie hielt aber keine Ressourcen vor für die intersubjektive Bearbeitung der Strittigkeit neuer wissenschaftlicher Erkenntnis. Um der präzisen Bearbeitung dieses vollständig neuen Zweckbereichs willen, so meine These, wichen die Pioniere der frühen Naturwissenschaft auf die Vernakulärsprachen aus, da sie dort für diesen Zweckbereich gesamtgesellschaftlich vorgehaltene Ressourcen antrafen.
} 


\author{
Anmerkung: scholion \\ Aufgabe: problema \\ Ausnahme: exceptio \\ Aussage: enunciatio \\ Begriff: notio, idea \\ Eigenschaft: attributum \\ Einbildungs-Kraft: imaginatio \\ Erklärung der Sache: definitio realis \\ Wort-Erklärung: definitio nominalis \\ Grund-Satz: axioma \\ Lehr-Satz: theorema \\ (Wolff 1726, zitiert nach Ricken 1995: 61)
}

Es ist deutlich, dass diese Ausdrücke nach zwei für das Deutsche fundamentalen Wortbildungsprinzipien entstanden sind: Wörter wie Einbildungskraft, Grundsatz und Lehrsatz sind Determinativkomposita. Und Wörter wie Anmerkung, Aussage und Begriff sind deverbale Ableitungen.

Diese Ausdrücke sind im Sinne von Gauger (1971) durchsichtig, indem das durch sie Benannte durch sie erhellt wird. Wolff hat hiermit eine terminologische Tradition der motivierten Benennung (Ricken 1995) begründet, der die deutschsprachige Philosophie und Wissenschaft verpflichtet bleiben. Betrachten wir hierzu einmal die Wortbildungen in dem folgenden Text Hegels (Kursivierungen i. O., Hervorhebungen im Fettdruck W.T.):

Nicht nur muß die Philosophie mit der Natur-Erfahrung übereinstimmend seyn, sondern die Entstehung und Bildung der philosophischen Wissenschaft hat die empirische Physik zur Voraussetzung und Bedingung. Ein anderes aber ist der Gang des Entstehens und die Vorarbeiten einer Wissenschaft, ein anderes die Wissenschaft selbst; in dieser können jene nicht mehr als Grundlage erscheinen, welche hier vielmehr die Nothwendigkeit des Begriffs seyn soll. - Es ist schon erinnert worden, daß außerdem der Gegenstand nach seiner Begriffsbestimmung in dem philosophischen Gange anzugeben ist, noch weiter die empirische Erscheinung, welche derselben entspricht, nahmhaft zu machen und von ihr aufzuzeigen ist, daß sie jener in der That entspricht. Diß ist jedoch in Beziehung auf die Nothwendigkeit des Inhalts kein Berufen auf die Erfahrung. (Hegel 1830: 236)

- deverbale Ableitungen: Entstehung, Bildung, Voraussetzung, Bedingung, Gang, Entstehen, Vorarbeit, Grundlage, Begriff, Beziehung, Berufen, Inhalt

- feldinterne Transpositionen: Entstehen, Berufen

- Rektionskomposita: Natur-Erfahrung, Begriffsbestimmung (mit deverbalen Ableitungen als Grundwort)

- desubstantivische Ableitung: Wissenschaft

- deadjektivische Ableitung: Nothwendigkeit

Fast die gesamten Substantive dieses Textes sind Wortbildungen, wobei den deverbalen Abstrakta die größte Bedeutung zukommt. Auch diese sind genushaltige Ausdrücke: Ableitungssuffixe wie -ung neutralisieren Verbalstämme hinsichtlich ihrer prototypischen Fusionsmöglichkeiten (entsteh-t), kommunizieren, wie das durch den Verbalstamm Benannte begrifflich zu aktualisieren ist (z.B. als nomen acti), und statten die resultierende Wortform mit Genus aus. 
Zusammenfassend: Der wissenschaftliche Sprachausbau des Englischen erfolgt im Zusammenhang der Erfordernisse der neuen, empirischen Naturwissenschaft. Um die Strittigkeit des neuen Wissens intersubjektiv bearbeiten zu können, bedienten sich die Naturwissenschaftler zunehmend der Vernakulärsprachen, in denen die sprachlichen Ressourcen für diese Erfordernisse gesamtgesellschaftlich vorgehalten wurden. Das scholastische Latein war für diese eristischen Erfordernisse so schlecht ausgebaut, dass z.B. die lateinische Übersetzung der $O p$ ticks in diesen Bereichen stark sinnentstellend ist. Der Ausbau des Englischen zur Wissenschaftssprache ereignet sich mithin im wesentlichen im eristischen Bereich, indem gemeinsprachliche Mittel für die Zwecke des wissenschaftlichen Streitens in den Dienst genommen werden. In terminologischer Hinsicht ist hingegen eine Kontinuität zu beobachten: die - bereits vorhandene - lateinische Terminologie wird im wesentlichen übernommen. Mit dem deutschen wissenschaftlichen Sprachausbau verhält es sich genau anders herum: Er ereignete sich im Rahmen der deutschen Aufklärung und sein Gegenstand war zunächst ganz wesentlich die Schaffung einer deutschen Terminologie. Hierfür werden vor allem zwei zentrale Wortbildungsverfahren einschlägig: die Komposition und die deverbale Ableitung. Beide Verfahren ermöglichen es, mit gemeinsprachlichen Mitteln eine Terminologie aufzubauen, die "durchsichtig" ist, indem sie das Benannte erhellt.

Für den Ausbau beider Sprachen zu Wissenschaftssprachen sind mithin vor allem die gemeinsprachlichen Ressourcen einschlägig. Diese kommen aber zunächst in völlig verschiedenen wissenschaftlichen Zweckbereichen zum Einsatz: im Englischen zur Ausbildung einer hochdifferenzierten Eristik, im Deutschen zunächst zur Ausbildung einer Terminologie, die den gnoseologischen Funktionsbereich im Rahmen wissenschaftlicher Anforderungen bedienen kann.

\section{Sprachtyp und wissenschaftlicher Sprachausbau - Konsequenzen für den gnoseo- logischen Funktionsbereich}

Aus den bisherigen Überlegungen zu den sprachtypologischen Unterschieden zwischen dem Englischen und Deutschen sowie den Differenzen im wissenschaftlichen Sprachausbau lassen sich folgende Schlüsse hinsichtlich des gnoseologischen Funktionsbereichs ziehen:

- Im Englischen stehen zur Bennennung begrifflicher Wissenskomplexe im wesentlichen pure Symbolfeldausdrücke (Redder 2005) zur Verfügung, deren begriffliche Nennqualität häufig über ihnen äußerliche Verfahren (z.B. Determination, Wortstellung) hergestellt wird. Dies betrifft auch die - z.B. aus dem Lateinischen entlehnte Terminologie. Im Lateinischen genushaltige Ableitungen (z.B. dispositio, refrangibilitas etc.) kommen als in ihrer Struktur nicht durchsichtige, pure Symbolfeldausdrücke zum Einsatz (disposition, refrangibility). ${ }^{5} \mathrm{Im}$ Englischen wird also zur Benennung wissenschaftlicher Begriffe zunächst im wesentlichen auf "vorfindliche", vorzugsweise aus dem Lateinischen stammende, Ausdrücke zurückgegriffen.

- Im Deutschen haben genushaltige Ausdrücke per se begriffliche Nennqualität. Die durch Wolff begründete Tradition der motivierten Terminologiebildung hat zur Konsequenz, dass für den Zweck der Benennung wissenschaftlicher Begriffe mit gemeinsprachlichen Mitteln eigene genushaltige Ausdrücke geschaffen werden, die im Hinblick auf das Benannte durchsichtig sind.

Zusammenfassend: Die Tatsache, dass das Englische die tradierte lateinische Terminologie im wesentlichen übernehmen konnte, während sie im Deutschen neu geschaffen werden musste, führt, in Verbindung mit den unterschiedlichen sprachtypologischen Voraussetzun-

\footnotetext{
5 Vgl. hierzu „Using these criteria, we could have dispositioned 869 cases as ineligible and saved one-third of the calls made to these cases, increasing the response rate by 5 percent." (Internet-Beleg)
} 
gen, zu fast diametral entgegengesetzten Verfahren der Begriffsbenennung im wissenschaftlichen Bereich: im Deutschen zu einer Tradition motivierter Benennung durch eigens aus gemeinsprachlichen Mitteln aufgebaute Ausdrücke von substantivischer Nennqualität; im Englischen zu einer Tradition der Entlehnung oftmals substantivischer Ausdrücke vorzugsweise aus dem Lateinischen, die in der isolierenden Sprache zu puren Symbolfeldausdrücken werden, deren begriffliche Nennqualität mit ihnen äußerlichen sprachlichen Verfahren herzustellen ist.

Die Konsequenzen für den gnoseologischen Funktionsbereich von Wissenschaftssprache sollen im folgenden an zwei Paralleltexten exemplarisch erörtert werden. ${ }^{6}$

\section{Benennung des wissenschaftlichen Erkenntnisgegenstands - Bearbeitung ein- und desselben Zwecks mit verschiedenen Mitteln}

Wissenschaftliche Praxis ist keine Elementarpraxis, sondern eine spezielle, eine abgeleitete Praxis, die bestimmte Verfahren der Elementarpraxis in den Dienst neugiergeleiteten Fragens stellt. ${ }^{7}$ Hierbei wird stets Neues einem Bekannten, nämlich dem wissenschaftlichen Erkenntnisgegenstand, zugesprochen. Dieses Bekannte ist aber, als wissenschaftlicher Erkenntnisgegenstand, zugleich der Elementarpraxis entrückt.

Der wissenschaftliche Erkenntnisgegenstand muss auf eine Weise benannt werden können, die ihn für den Zuspruch neuen Wissens offenhält. Dies bedeutet, dass die Benennung möglichst wenig ontologisches Eigengewicht aufweisen sollte. Zugleich soll die Benennung aber auch unauffällig genug sein, so dass sie hinter dem Neuen zurücktreten kann.

Die symbolischen Ausdrücke, die diese erhebliche Funktionslast tragen, sind die "Nahtstellen" zwischen bekanntem und neuem Wissen. Mit ihnen wird der wissenschaftliche Erkenntnisgegenstand hantierbar gemacht, sowohl sprachlich-operativ als auch begrifflich. Sie müssen daher sowohl mit innersprachlichen Gegebenheiten und Zwecken kompatibel sein, als auch den Anforderungen an adäquate und sinnvolle Begriffsbenennung genügen. Sie sind für die gnoseologische Funktion von Wissenschaftssprache besonders einschlägig.

Der Erkenntnisgegenstand in den beiden hier exemplarisch untersuchten wissenschaftlichen Aufsätzen aus der Phonetik ist Intonation. Ich betrachte zunächst exemplarisch sechs Belege aus dem englischen Aufsatz "Phonological Features of Intonational Peaks" (Ladd 1983, Hervorhebungen im Fettdruck W.T.):

\footnotetext{
6 Vgl. hierzu auch Thielmann (2009: 278-282).

7 Vgl. hierzu auch (Mittelstraß 1974, 65f.): "Die Korrektur der Annahme, der Erfahrungsbegriff einer vortheoretischen Lebenspraxis bilde nicht nur die Basis, sondern auch den Begründungsrahmen einer empirischen Wissenschaft, erfolgt im Rahmen der Galileischen Physik durch die Einführung des Experiments als einer, wie sich jetzt sagen läßt, technisch kontrollierten 'neuen' Erfahrung. An die Stelle der vor-theoretischen Lebenspraxis, die in allen ihren Wesentlichen Teilen stets eine elementare Unterscheidungs- und Orientierungspraxis ist, tritt als eine unter bestimmten Zielen eingeschränkte, ursprünglich ebenfalls dem vortheoretischen Bereich zugehörige Herstellungspraxis die technische Praxis. In dieser technischen Praxis wird unter der Leitung theoretischer Fragestellungen, die, wie nicht anders zu erwarten, noch ganz den Aristotelischen Erwartungen entsprechen, Erfahrung instrumental erzeugt. Der Galileische Erfahrungsbegriff ist damit im Unterschied zum Aristotelischen Erfahrungsbegriff von vornherein an die Bedingungen einer messenden Physik gebunden; als empirisches Wissen tritt nicht mehr auf, was sich als ein vor-theoretisches Wissen theoretisch fassen läßt, sondern was mit den Instrumentarien einer physikalischen bzw. technischen Praxis (häufig gegen das Erfahrungswissen einer lebensweltlichen Praxis) gewonnen wurde. Daher ändert sich mit dem Begriff der Erfahrung zugleich der Begriff des Empirischen bzw. der Begriff dessen, was empirisch begründet heißen darf. Als 'empirisch' oder 'empirisch begründet' kann im Rahmen des Galileischen, für die Methodologie der Physik in Geltung bleibenden Erfahrungsbegriffs nur noch das Ergebnis einer messenden Praxis bezeichnet werden."
} 
(12) This paper introduces and illustrates a model of intonation in which structurally significant points of contours are specified by phonological features. (721)

(13) In particular, it borrows from them the idea that contours can be represented as sequences of tones associated with accents and boundaries, and the concept of a primary phonological opposition of pitch between high and low. (721)

(14) In this view, the basic units of intonation (at least in the European languages) are taken to be phrase- or utterance-level contours: approximate over-all shapes that are correlated with grammatical meanings like 'declarative' and 'continuation'. (722)

(15) While Pierrehumbert and 't Hart avoid many of the problems inherent in the CI approach, they have trouble expressing generalizations based on over-all contour shape. (723)

(16) Second, there is no way to express meaningful distinctions in the 'over-all slope' of contours, i.e. differences in the slope of lines drawn through the accent-related peaks or valleys. (723)

(17) Similarly, P talks about a family of contours in English characterized by a rather steep over-all downward trend over accent peaks, where 'rather steep' is understood to mean 'steeper than would be expected on the basis of declination' (cf. fn. 1, above). (724)

Die materielle Gegebenheit der $\mathrm{F}_{0}$-Variation als Grundlage der phonologischen Interpretation wird in dem englischen Text durchgehend mit dem Ausdruck contour benannt (insgesamt 145 Vorkommen auf 34 Seiten). Contour wurde im 17. Jh. als Fachausdruck der bildenden Künste aus dem Französischen entlehnt; der Ausdruck wird seit dem 19. Jh. auch als Prädikat eingesetzt (OED). Contour ist also synchron ein purer Symbolfeldausdruck, der durch seine Einbettung in die syntaktischen "Slots" des Subjekts (13), Prädikativs (14) und Präpositionalattributs ((12), (16), (17) eine kategoriale Ausdeutung des durch ihn benannten Gehalts als begriffliche Wissensstruktur nahelegt. In dem Syntagma over-all contour shape (15) findet eine solche kategoriale Vereindeutigung hingegen nicht statt.

Inhaltlich hat man es mit einer Metapher zu tun: Von der ursprünglichen Bedeutung "Umrisslinie"/"umreißen" verbleiben die Aspekte der Linie und der Form; "umrissen", im Sinne bildlicher Darstellung, wird hingegen nichts mehr. Mit contour wird also die Abbildung der $\mathrm{F}_{0^{-}}$ Variation ohne Reflexion ihrer Abbildungsqualität erfasst. Intonation, als zeitlicher Ablauf, ist mithin nur noch in hypostasierter Form zugänglich. Dies zeigt sich auch daran, wie mit contour im einzelnen operiert wird:

Die structurally significant points of contours (12) sind Elemente des shape ((14), (15)), das sowohl die Feststellung eines over-all slope (16) (wie bei einer mathematischen Funktion) als auch die Etablierung einer family of contours (17) (durchaus im Sinne einer Funktionenschar) gestattet. Auch die sequences of tones (13) sind von contour her gedacht.

Ich betrachte nun die Verhältnisse im Deutschen anhand einiger Belege aus dem Text "Intonation und Fokus im Hamburgischen" (Peters 2002, Hervorhebungen W.T.):

(18) Die $\boldsymbol{F}_{0}$-Verläufe in Abb. 4 zeigen, dass der $\boldsymbol{F}_{0}$-Gipfel bei weitem Fokus im Bereich der 1. Hälfte des Nukleus erreicht wird, bei engem Fokus in der 2. Hälfte des Nukleus. (34)

(19) Die $\boldsymbol{F}_{\boldsymbol{0}}$-Verläufe bei weitem und engem Fokus unterscheiden sich hier in erster Linie in der Geschwindigkeit der terminalen Fallbewegung von $\boldsymbol{F}_{0}$. (38) 
(20) Der Verlauf der postnuklearen Fallbewegung von $F_{0}$ im Hamburgischen spricht allerdings eher gegen einen bitonalen Akzent, denn die Fallbewegung erreicht die Grundlinie bei nicht-finalen Akzentsilben meist erst am Ende der IP oder an der hinteren Grenze des Fußes, in dem die Fokusakzentsilbe auftritt, d.h. in jedem Falle an einer Phrasengrenze. (49)

(21) Die Konturen in Abb. 3 zeigen für die Beispiele in (8), dass der Gipfel bei weitem Fokus im Bereich des Kurzvokals auftritt, bei engem Fokus im Bereich des CodaSonoranten. (34)

(22) Ladd (1983) trat für die Charakterisierung tonaler Einheiten mittels zusätzlicher Merkmale wie [ \pm delay] oder [ \pm downstep] ein, um der Ähnlichkeit zwischen Konturen, die sich nur hinsichtlich einer einzigen Merkmalsdimension unterscheiden, Rechnung zu tragen und nicht gänzlich unterschiedliche tonale Strukturen ansetzen zu müssen. (47)

(23) Die Assoziationsbeziehungen der Töne legen die Ausrichtung der Akzentgipfel nicht genau fest, sie schränken jedoch die zeitliche Realisierung der Intonationskontur ein: Unterschiedliche Assoziationsbeziehungen führen zu einer unterschiedlichen zeitlichen Gestaltung der Kontur, sofern nicht besondere Realisationsbedingungen, z.B. das Fehlen geeigneten segmentalen Materials, zu einer Neutralisierung des Unterschieds auf der phonetischen Ebene führen. (47)

Auch im Deutschen wird der Ausdruck Kontur genutzt ((21)-(23)), dessen Entlehnungsgeschichte der Situation im Englischen vergleichbar ist. Darüber hinaus wird ein weiterer Ausdruck zur Konzeptualisierung der materiellen Gegebenheit der $\mathrm{F}_{0}$-Variation eingesetzt, bei dem es sich um ein deverbales Abstraktum handelt: der Ausdruck Verlauf ((18)-(20)).

Im Rahmen der traditionellen Wortbildungslehre ist die Einordnung von Ausdrücken wie Lauf nicht unproblematisch: Im Gegensatz zu sog. impliziten Ableitungen wie Wurf, Schuss und Zug weisen sie keinen Vokalwechsel auf, so dass die Klassifizierungen zwischen "Konvertat" (Donalies 2002, 129) und "paradigmatischer Umsetzung" (Erben 2004) schwanken (vgl. hierzu auch Eisenberg 2004 Bd.1: 294-300). Im Rahmen der eingangs vorgetragenen Überlegungen zu Symbolfeldausdrücken und ihrer Funktion betrachte ich Lauf als deverbale Ableitung, die aus dem genuslosen, nicht als freies Morphem realisierbaren Stamm -lauf- das Maskulinum Lauf erzeugt, das als Substantiv benennt.

Die deverbale Ableitung Verlauf macht zeitlich Veränderliches begrifflich verfügbar. Auf das Intonationsphänomen angewandt, bedeutet dies, dass Intonation hinsichtlich ihrer Prozessualität zugänglich wird. Dies sieht man auch ganz deutlich, wenn man sich das jeweils dem Erkenntnisgegenstand zugesprochene neue Wissen ansieht:

Obwohl in (18) mit dem fachsprachlichen Plural Verläufe auf graphische Darstellungen von Intonationskurven Bezug genommen wird, "wird der $F_{0}$-Gipfel bei weitem Fokus im Bereich der 1. Hälfte des Nukleus erreicht" und "liegt" nicht etwa "im Bereich der 1. Hälfte des Nukleus". In (19) ist von der "Geschwindigkeit der terminalen Fallbewegung von $F_{0}$ " die Rede, in (20) davon, dass die "Fallbewegung die Grundlinie erreicht". Es dominiert das Bewegungskonzept.

Wo es um "Konturen" geht, werden keine Gipfel erreicht, sondern sie "treten auf" (21). In (22) wird Ladd (1983) zitiert und hinsichtlich seiner statischen Konzeptionen reproduziert: "Ähnlichkeit zwischen Konturen, die sich nur hinsichtlich einer einzigen Merkmalsdimension unterscheiden".

Sehr interessant ist der Beleg (23): Mehrere empirisch als typisch erwiesene "Verläufe" fügen sich zu einer abstrakten "Kontur", deren "zeitliche Realisierung/Gestaltung" von bestimmten 
Bedingungen abhängt. Hier ist der statische Kontur-Begriff durch die "Verlaufs-Perspektive" informiert.

Es ist also zu beobachten, dass es im deutschen Text zu zwei unterschiedlichen, einander ergänzenden Konstitutionen des Erkenntnisgegenstandes kommt: Der Konturbegriff gestattet die Feststellung von Merkmalen, die Ermittlung von Typischem. Der Verlaufs-Begriff gestattet eine phänomenangemessene Erfassung der zeitlichen Intonationsentwicklung.

Zusammenfassend: Die begriffliche Konstituierung des Erkenntnisgegenstandes ist, wenn man es mit zeitlichen Abläufen zu tun hat, wissenschaftssprachlich eine besonders anspruchsvolle Aufgabe. Wie am Beispiel der Konzeptualisierung des Intonationsphänomens für die Zwecke phonologischer Analyse gezeigt werden konnte, bestehen in diesem Bereich zwischen dem Englischen und dem Deutschen systematische Differenzen, die von den sprachtypologischen Unterschieden zwischen den beiden Sprachen herrühren.

Da Wortbildung im Englischen sehr eingeschränkt ist, ist der Wissenschaftler gezwungen, zur begrifflichen Konstituierung des Erkenntnisgegenstandes aus dem Bestand an vorliegenden Wörtern eine symbolische Prozedur auszuwählen, deren Benanntes "möglichst gut" mit den von ihm verfolgten Zwecken harmoniert. Gerade mit einer besonders geglückten Wahl, wie sie die Metapher contour für das Englische zweifelsohne darstellt, kann jedoch eine Festlegung verbunden sein, die den Blick auf bestimmte Aspekte des Gegenstandsbereichs verstellt: Wird Intonation, also ein Ablauf in der Zeit, ausschließlich als contour vergegenständlicht, erfolgt eine Festlegung auf geometrische Aspekte ihrer Abbildung, ohne dass die Abbildungstatsache noch in diesem Begriff reflektiert ist.

Der deutsche Text, der von der Kontur-Metapher ebenfalls Gebrauch macht, setzt darüber hinaus noch das deverbale Abstraktum Verlauf ein, das der zeitlichen Qualität des Untersuchungsgegenstandes Rechnung trägt. Es war zu beobachten, dass es hierdurch zu zwei verschiedenen, einander ergänzenden Konstituierungen des Erkenntnisgegenstandes kommt.

\section{$6 \quad$ Fazit}

Sprachen können hinsichtlich der Mittel, mit denen sie den gnoseologischen Funktionsbereich bedienen, stark differieren. Zwar werden hierfür vorwiegend sprachliche Mittel des Symbolfeldes relevant; diese können aber unterschiedlich realisiert sein. Wie deutlich wurde, hält das Deutsche für die Fixierung und Kommunikation begrifflicher Wissenskomplexe eine durch Genus ausgezeichnete Ausdrucksklasse vor: die Substantive, die prototypisch als Köpfe von Nominalphrasen zum Einsatz kommen. Das Englische verfügt über eine solche Ausdrucksklasse nicht. Vielmehr liegen im Symbolfeld im wesentlichen pure Symbolfeldausdrücke im Sinne von Redder (2005) vor. Begriffliche Nennqualität wird durch diesen Ausdrücken äuBerliche Mittel (z.B. Determination, Attributionsverfahren etc.) erst hergestellt.

Vor dem Hintergrund dieser typologischen Differenzen sind die - selbst höchst verschiedenen - wissenschaftlichen Ausbaugeschichten beider Sprachen zu bewerten. Der wissenschaftliche Sprachausbau des Englischen ereignet sich ganz wesentlich im Bereich des wissenschaftlichen Streitens, der Eristik. Wie gezeigt werden konnte, wird dieser Bereich mit gesamtgesellschaftlich vorgehaltenen gemeinsprachlichen Mitteln bedient - ein Vorteil, um dessentwillen das Lateinische als Wissenschaftssprache aufgegeben wird. Im gnoseologischen Funktionsbereich herrscht hingegen eine Kontinuität: die lateinische Terminologie wird im wesentlichen übernommen. Lateinische Derivationen (deverbale und deadjektivische Abstrakta) werden hierbei zu puren Symbolfeldausdrücken, die auch hinsichtlich ihrer inneren, lateinischen Wortbildungsstruktur nicht mehr durchsichtig sind. Der Ausbau des Deutschen zur Wissenschaftssprache ereignet sich später und in diametral entgegengesetzter Weise: Er steht im Zeichen der Aufklärung; seine treibende Kraft ist nicht die Naturwissenschaft, sondern die Philo- 
sophie; sein wesentlicher Gegenstand ist die Terminologie. Wolff, der hier für die gesamte deutsche Wissenschaftstradition prägend geblieben ist, nutzt für den Aufbau deutscher Terminologie im wesentlichen zwei Wortbildungsverfahren: Determinativkomposition und deverbale Ableitung. Die so entstehenden Substantive sind aus gemeinsprachlichen Mitteln nach gemeinsprachlichen Verfahren gebildet und mithin durchsichtig, indem sie das durch sie Benannte erhellen. In beiden Sprachen werden mithin zwar gemeinsprachliche Mittel für den wissenschaftlichen Sprachausbau einschlägig. Diese werden aber in völlig unterschiedlichen Bereichen relevant. Im Englischen werden sie zum Aufbau einer hochdifferenzierten Eristik genutzt; im Deutschen hingegen zunächst zum Aufbau einer Terminologie im Rahmen der gnoseologischen Erfordernisse von Wissenschaft.

Wie ein Vergleich zweier Paralleltexte aus dem Bereich der Phonetik ergeben hat, prägen sich die mit Notwendigkeit differenten Lösungen im gnoseologischen Funktionsbereich entsprechend aus: Zur Benennung des wissenschaftlichen Erkenntnisgegenstandes wird im Englischen von einer Metapher Gebrauch gemacht: contour. Intonation, ein zeitlich veränderliches Phänomen, wird so hinsichtlich ihrer graphischen Umsetzung diskutierbar wie eine mathematische Funktion. Zugleich wird der Erkenntnisgegenstand um seine prozessuale Dimension reduziert. Im Deutschen werden sowohl die Kontur-Metapher als auch die deverbale Ableitung Verlauf verwendet, die, wie am Text ersichtlich, den Erkenntnisgegenstand hinsichtlich seiner geschichtlichen Dimension offenhält.

Die gnoseologischen Ressourcen der deutschen und englischen Wissenschaftssprache verhalten sich aufgrund sprachtypologischer Differenzen und unterschiedlicher eingeschlagener Wege beim wissenschaftlichen Sprachausbau alternativ zueinander. ${ }^{8}$ Dies ist ein zentrales Argument für den Erhalt wissenschaftlicher Mehrsprachigkeit im Interesse des wissenschaftlichen Erkenntnisfortschritts: Das in der gnoseologischen Differenz angelegte Innovationspotential sollte nicht einer sprachlichen Monokultur zum Opfer fallen.

\section{Literatur}

Blake, Norman (ed.) (1992): The Cambridge history of the English language. Vol. II: 10661476. Cambridge: CUP.

Bühler, Karl (1934 ND 1982): Sprachtheorie. Stuttgart: Fischer.

Burgess, Anthony (2000): A Clockwork Orange. London: Penguin.

Donalies, Elke (2002): Die Wortbildung des Deutschen. Ein Überblick. Tübingen: Narr.

Ehlich, Konrad (1981): Sprachmittel und Sprachzwecke. Antrittsvorlesung Universität Düsseldorf. (= 1982 als TILL 1 (Tilburg Papers in Language and Literature).

Ehlich, Konrad (1986): "Funktional-pragmatische Kommunikationsanalyse - Ziele und Verfahren". In: Hartung, Wolfdietrich (ed.): Untersuchungen zur Kommunikation - Ergebnisse und Perspektiven. Berlin, Akademie: 15-40.

Ehlich, Konrad (1993): "Deutsch als fremde Wissenschaftssprache". In: Jahrbuch Deutsch als Fremdsprache 19. München, iudicium: 13-42.

Ehlich, Konrad (1995): "Die Lehre der deutschen Wissenschaftssprache: sprachliche Strukturen, didaktische Desiderate". In: Kretzenbacher, Heinz L./Weinrich, Harald (eds.): Linguistik der Wissenschaftssprache. Berlin, de Gruyter: 325-352.

Ehlich, Konrad (1998 ND 2007): "Medium Sprache". In: Ders. (ed.): Sprache und sprachliches Handeln. Bd. 1. Pragmatik und Sprachtheorie. Berlin/New York, de Gruyter: 151165.

Eisenberg, Peter $\left(2004^{2}\right)$ : Grundriß der deutschen Grammatik. Bd. I: Das Wort. Stuttgart: Metzler.

\footnotetext{
${ }^{8}$ Dies betrifft die in Natur- und Geisteswissenschaften zum Einsatz kommenden sprachlichen Mittel gleichermaßen (vgl. auch Thielmann 2009).
} 
Eisenberg, Peter (2004²): Grundriß der deutschen Grammatik. Bd. II: Der Satz. Stuttgart, Metzler.

Erben, Johannes $\left(2000^{4}\right)$ : Einführung in die deutsche Wortbildungslehre. Berlin: ESV .

Gauger, Hans-Martin (1971): Durchsichtige Wörter. Zur Theorie der Wortbildung. Heidelberg: Winter.

Hegel, Georg W. F. (1830; 1992): Enzyklopädie der philosophischen Wissenschaften im Grundrisse. Hamburg: Meiner. (Gesammelte Werke hg. v. d. Rheinisch-Westfälischen Akademie der Wissenschaften 20).

Hoffmann, Ludger (2003): "Funktionale Syntax: Prinzipien und Prozeduren". In: Ders. (ed.): Funktionale Syntax. Die pragmatische Perspektive. Berlin/New York, de Gruyter: 18-121.

Hopkins, Gerard Manley (1961): Selected Poems. London: Heinemann.

Kuenkamp, Annette (1995): "Psychoanalyse ohne Seele? Zur englischen Übersetzung der psychoanalytischen Terminologie Sigmund Freuds". In: Kretzenbacher, Heinz L./Weinrich, Harald (eds.): Linguistik der Wissenschaftssprache. Berlin, de Gruyter: 121154.

Ladd, D. Robert (1983): "Phonological features of intonational peaks." Language 59/4: 721759.

Maier, Anneliese (1955): Metaphysische Hintergründe der spätscholastischen Naturphilosophie. Rom: Storia e letteratura.

Mittelstraß, Jürgen (1974) Die Möglichkeit von Wissenschaft. Frankfurt: Suhrkamp.

Newton, Isaac (172633; ND 1972): Philosophiae naturalis principia mathematica. Assembled and edited by Alexandre Koyré and I. Bernhard Cohen. Cambridge: University Press.

Newton, Isaac (1704): Opticks: or a Treatise of the Reflexions, Refractions, Inflexions and Colours of Light. London. http://www.rarebookroom.org/Control/nwtopt/index.html, Stand 31. 10. 2010.

Newton, Isaac (1706): Optice: Sive de Reflexionibus, Refractionibus, Inflexionibus \& Coloribus Lucis. London. http://echo.mpiwg-berlin.mpg.de/ECHOdocuView?url= $\% 2$ Fmpiwg\%2Fonline \%2Fpermanent $\% 2$ Flibrary\%2F4K35AAGC\%2Findex.meta\&start=1 $1 \&$ view Mode $=$ images $\& p n=18 \&$ mode $=$ texttool, Stand 31. 10. 2010.

Peters, Jörg (2002): "Intonation und Fokus im Hamburgischen." Linguistische Berichte 189: 27-57.

Redder, Angelika (2005): "Wortarten oder sprachliche Felder, Wortartenwechsel oder Feldtransposition?" In: Knobloch, Clemens/Schaeder, Burkhard (eds.): Wortarten und Grammatikalisierung. Perspektiven in System und Erwerb. Berlin/New York, de Gruyter: 43-66.

Ricken, Ulrich (1995): "Zum Thema Christian Wolff und die Wissenschaftssprache der deutschen Aufklärung". In: Kretzenbacher, Heinz L./Weinrich, Harald (eds.): Linguistik der Wissenschaftssprache. Berlin, de Gruyter: 41-90.

Simonyi, Karoly (1990): Kulturgeschichte der Physik. Budapest: Athenaeum Nyomda.

Thielmann, Winfried (1999): Fachsprache der Physik als begriffliches Instrumentarium exemplarische Untersuchungen zur Funktionalität naturwissenschaftlicher Begrifflichkeit bei der Wissensgewinnung und -strukturierung im Rahmen der experimentellen Befragung von Natur. Frankfurt/M: Lang.

Thielmann, Winfried (2004): "Begriffe als Handlungspotentiale." Linguistische Berichte 199: 287-311.

Thielmann, Winfried (2009): Deutsche und englische Wissenschaftssprache im Vergleich: Hinführen - Verknüpfen - Benennen. Heidelberg: Synchron.

Thielmann, Winfried (2010): "Wege aus dem sprachpolitischen Vakuum? Zur scheinbaren wissenschaftskulturellen Neutralität wissenschaftlicher Universalsprachen". In: Ehlich, Konrad (ed.): Mehrsprachige Wissenschaft. Heidelberg: Synchron [im Druck; abrufbar unter www.euro-sprachenjahr.de/Thielmann.pdf]. 
Vogel, Petra M. (2000): "Grammaticalisation and part-of-speech systems". In: Vogel, Petra M./Comrie, Bernard (eds.): Approaches to the Typology of Word Classes. Berlin/New York, de Gruyter: 259-284.

Weinrich, Harald $\left(2003^{2}\right)$ : Textgrammatik der deutschen Sprache. Hildesheim: Olms.

Zifonun, Gisela/Hoffman, Ludger/Strecker, Bodo et al. (1997): Grammatik der deutschen Sprache. Berlin/New York: de Gruyter. 